\title{
On the average number of divisors of the sum of digits of squares
}

\author{
Teerapat Srichan \\ Department of Mathematics, Faculty of Science \\ Kasetsart University, Bangkok, Thailand \\ e-mail: fscitrpaku.ac.th
}

\begin{abstract}
For a positive integer $q>1$, we present the estimation of the average number of number of divisors of the sum of digits of squares. Moreover, we extend the result to the sum of digits of the power $h, h \geq 2$.
\end{abstract}

Keywords: Divisor function, Sum of digits.

2010 Mathematics Subject Classification: 11N37, 11N69.

\section{Introduction and statement of the main results}

Let $q \geq 2$ be an integer. For $n \in \mathbb{N}$ can be written uniquely in base $q$ as

$$
n=\sum_{k=0}^{\infty} a_{k}(n) q^{k}, \quad a_{k}(n) \in\{0,1, \ldots, q-1\} .
$$

For a fixed $q$, the sum of digits of $n$ in base $q$ is defined by

$$
s_{q}(n)=\sum_{k=0}^{\infty} a_{k}(n)
$$

The function $s_{q}(n)$ occurs in many branches of mathematics. We refer the reader to the monograph [7] (see Chapter IV, part 4.3) for more details. Let $d(n)$ denote the number of positive divisors of the integer $n$. There are many research on the determining the asymptotic behaviour of the divisor sum 


$$
\sum_{n \leq x} d(f(n)) \quad \text { for } f(x) \text { is a given polynomial. }
$$

For example, in 1952, Erdös [1] showed that for an irreducible polynomial $P(x) \in \mathbb{Z}[x]$ and for any $N>1$,

$$
N \log N \ll_{P} \sum_{n \leq N} d(P(n)) \ll_{P} N \log N
$$

where the dependence of the constants can be both on the degree and the coefficients of the polynomial $P(x)$. Next, Hooley [4] proved that

$$
\sum_{n \leq N} d\left(n^{2}+a\right)=c_{1} N \log N+c_{2} N+O\left(N^{8 / 9} \log ^{3} N\right)
$$

for any fixed $a \in \mathbb{Z}$ such that $-a$ is not a perfect square, where $c_{1}$ and $c_{2}$ are constants depending only on $a$. For other results related to the sum in (1.1), we refer the reader to [5] and [8] (see Chapter II) for more details.

In this paper, we shall determine the asymptotic behaviour of the divisor sum, which is similar as in (1.1) related to the sum of digits of $n$ in base $q$. Here are our main results.

Theorem 1.1. For $N, q \geq 2$, and $q-1=\prod_{i=1}^{r} p_{i}^{s_{i}}$. Then, for a small $\varepsilon>0$, we have

$$
\begin{aligned}
\sum_{n \leq N} d\left(s_{q}\left(n^{2}\right)\right) & =\frac{1+\ln \left((q-1)\left\lfloor\log _{q} q N\right\rfloor\right)}{q-1} \prod_{i=1}^{r}\left(p_{i}^{\left\lfloor\frac{s_{i}}{2}\right\rfloor}+\left(p_{i}-1\right) \sum_{b=1}^{s_{i}} p_{i}^{\left\lfloor\frac{s_{i}+b}{2}\right\rfloor-1}\right) N \\
& +O_{q}\left(N(\log N)^{-1+\varepsilon}\right) .
\end{aligned}
$$

Moreover, we extend Theorem 1.1 to the sum of digits of the power $h, h \geq 2$.

Theorem 1.2. For $N, h \geq 2$, and $q \geq \exp \left(67 h^{3}(\log h)^{2}\right)$ be a sufficiently large prime number such that $q-1=\prod_{i=1}^{r} p_{i}^{s_{i}}$. Then, for a small $\varepsilon>0$, we have

$$
\begin{aligned}
\sum_{n \leq N} d\left(s_{q}\left(n^{h}\right)\right)= & \frac{1+\ln \left((q-1)\left\lfloor\log _{q} q N\right\rfloor\right)}{q-1} \prod_{i=1}^{r}\left(p_{i}^{\left\lfloor\frac{s_{i}(h-1)}{h}\right\rfloor}+\left(p_{i}-1\right) \sum_{b=1}^{s_{i}} p_{i}^{\left\lfloor\frac{s_{i}+b}{h}\right\rfloor-1}\right) N \\
& +O_{q}\left(N(\log N)^{-1+\varepsilon}\right) .
\end{aligned}
$$

\section{Lemmas}

The following lemmas will be used in the proof of (1.2) and (1.3).

Lemma 2.1. [6, Theorem 3] For any integers $q \geq 2$ and $m \geq 2$. There exists $\sigma_{q, m}>0$ such that

$$
\#\left\{n \leq x: s_{q}\left(n^{2}\right) \equiv 0 \bmod m\right\}=\frac{x}{m} Q(0, D)+O\left(x^{1-\sigma_{q, m}}\right),
$$

where $D=\operatorname{gcd}(q-1, m)$ and $Q(0, D)=\#\left\{0 \leq n<D: n^{2} \equiv 0 \bmod D\right\}$. 
Lemma 2.2. [3, Corollary 5] Given a multiplicative function $f$, the sum over $k, k=1, \ldots, n$ of the function values $f(\operatorname{gcd}(k, n))$ of the greatest common divisor can be expressed in the prime factors of the number $n=\prod_{i=1}^{r} p_{i}^{s_{i}}$ as

$$
\sum_{k=1}^{n} f(\operatorname{gcd}(k, n))=\prod_{i=1}^{r}\left(f\left(p_{i}^{s_{i}}\right)+\left(p_{i}-1\right) \sum_{b=1}^{s_{i}} p_{i}^{b-1} f\left(p_{i}^{s_{i}-b}\right)\right) .
$$

For $x \in \mathbb{R} / \mathbb{Z}, \Psi(x)=x-\lfloor x\rfloor-\frac{1}{2}$.

Lemma 2.3. For $T>1$, if $q-1 \backslash T$, then

$$
\begin{aligned}
\sum_{d=1}^{T} Q(0, \operatorname{gcd}(q-1, d))= & \frac{T}{q-1} \prod_{i=1}^{r}\left(p_{i}^{\left\lfloor\frac{s_{i}}{2}\right\rfloor}+\left(p_{i}-1\right) \sum_{b=1}^{s_{i}} p_{i}^{\left\lfloor\frac{s_{i}+b}{2}\right\rfloor-1}\right) \\
& +O_{q}\left(\Psi\left(\frac{T}{q-1}\right)\right) .
\end{aligned}
$$

If $q-1 \mid T$, then

$$
\sum_{d=1}^{T} Q(0, \operatorname{gcd}(q-1, d))=\frac{T}{q-1} \prod_{i=1}^{r}\left(p_{i}^{\left\lfloor\frac{s_{i}}{2}\right\rfloor}+\left(p_{i}-1\right) \sum_{b=1}^{s_{i}} p_{i}^{\left\lfloor\frac{s_{i}+b}{2}\right\rfloor-1}\right) .
$$

Proof. Let $q-1=\prod_{i=1}^{r} p_{i}^{s_{i}}$. For $q=2$, the empty product is 1 . Thus

$$
\sum_{d=1}^{T} Q(0, \operatorname{gcd}(1, d))=\sum_{d=1}^{T} Q(0,1)=\sum_{d=1}^{T} 1=T
$$

Now, we consider $q>2$. For $q-1 \mid T$, we have the result (2.4) by applying Lemma 2.2. In order to apply Lemma 2.2, we need to show that the arithmetical function $Q(0, \operatorname{gcd}(q-1,)$.$) is$ multiplicative. It is trivial that $Q(0, \operatorname{gcd}(q-1,1))=1$ and for any prime number $p$, and nonnegative integer $\alpha$, we have $Q\left(0, \operatorname{gcd}\left(q-1, p^{\alpha}\right)\right)=Q\left(0, p^{\alpha^{\prime}}\right)=p^{\left\lfloor\frac{\alpha^{\prime}}{2}\right\rfloor}$, where $\operatorname{gcd}\left(q-1, p^{\alpha}\right)=p^{\alpha^{\prime}}$. Then, for a product of prime $p_{i}, i=1, \ldots, k$,

$$
\left.Q\left(0, \operatorname{gcd}\left(q-1, \prod_{i=1}^{k} p_{i}^{\alpha_{i}}\right)\right)=Q\left(0, \prod_{i=1}^{k} p_{i}^{\alpha_{i}^{\prime}}\right)\right)=\prod_{i=1}^{k} p_{i}^{\left\lfloor\frac{\alpha_{i}^{\prime}}{2}\right\rfloor}=\prod_{i=1}^{k} Q\left(0, \operatorname{gcd}\left(q-1, p_{i}^{\alpha_{i}^{\prime}}\right)\right)
$$

This shows the multiplication property of the function $Q(0, \operatorname{gcd}(q-1,)$.$) .$

Thus, in view of Lemma 2.2, we have

$$
\sum_{d=1}^{q-1} Q(0, \operatorname{gcd}(d, q-1))=\prod_{i=1}^{r}\left(p_{i}^{\left\lfloor\frac{s_{i}}{2}\right\rfloor}+\left(p_{i}-1\right) \sum_{b=1}^{s_{i}} p_{i}^{\left\lfloor\frac{s_{i}+b}{2}\right\rfloor-1}\right) .
$$

Since the function $Q(0, \operatorname{gcd}(q-1,)$.$) is periodic with period q-1$, thus, for $q-1 \mid T$

$$
\sum_{d=1}^{T} Q(0, \operatorname{gcd}(q-1, d))=\frac{T}{q-1} \prod_{i=1}^{r}\left(p_{i}^{\left\lfloor\frac{s_{i}}{2}\right\rfloor}+\left(p_{i}-1\right) \sum_{b=1}^{s_{i}} p_{i}^{\left\lfloor\frac{s_{i}+b}{2}\right\rfloor-1}\right)
$$


For $q-1 \nmid T$, we have

$$
\begin{aligned}
\sum_{d=1}^{T} Q(0, \operatorname{gcd}(q-1, d))= & \sum_{d=1}^{q-1} Q(0, \operatorname{gcd}(q-1, d))+\cdots+\sum_{d=\left\lfloor\frac{T}{q-1}\right\rfloor(q-1)+1}^{T} Q(0, \operatorname{gcd}(q-1, d)) \\
= & \left\lfloor\frac{T}{q-1}\right\rfloor \prod_{i=1}^{r}\left(p_{i}^{\left\lfloor\frac{s_{i}}{2}\right\rfloor}+\left(p_{i}-1\right) \sum_{b=1}^{s_{i}} p_{i}^{\left\lfloor\frac{s_{i}+b}{2}\right\rfloor-1}\right) \\
& +\sum_{d=\left\lfloor\frac{T}{q-1}\right\rfloor(q-1)+1}^{T} Q(0, \operatorname{gcd}(q-1, d)) \\
= & \left\lfloor\frac{T}{q-1}\right\rfloor \prod_{i=1}^{r}\left(p_{i}^{\left\lfloor\frac{s_{i}}{2}\right\rfloor}+\left(p_{i}-1\right) \sum_{b=1}^{s_{i}} p_{i}^{\left\lfloor\frac{s_{i}+b}{2}\right\rfloor-1}\right) \\
& +\left(T-\left\lfloor\frac{T}{q-1}\right\rfloor(q-1)\right) \\
& +\quad \sum_{d=\left\lfloor\frac{T}{q-1}\right\rfloor(q-1)+1}^{T}(Q(0, \operatorname{gcd}(q-1, d))-1) .
\end{aligned}
$$

Now we bound the last term.

$$
\begin{aligned}
& \sum_{\substack{d=\left\lfloor\frac{T}{q-1}\right\rfloor(q-1)+1 \\
\operatorname{gcd}(q-1, d) \text { is not a squarefree }}}^{T} Q(0, \operatorname{gcd}(q-1, d)) \\
& \leq \#\left\{n \mid\left\lfloor\frac{T}{q-1}\right\rfloor(q-1)<n p^{2} \leq T, \text { for all } p \mid(q-1)\right\} \prod_{i=1}^{r} p_{i}^{\left\lfloor\frac{s_{i}}{2}\right\rfloor} \\
& \leq\left(T-\left\lfloor\frac{T}{q-1}\right\rfloor(q-1)\right) \prod_{i=1}^{r} p_{i}^{\left\lfloor\frac{s_{i}}{2}\right\rfloor} \sum_{p_{i}} \frac{1}{p_{i}^{2}} \\
&=O_{q}\left(T-\left\lfloor\frac{T}{q-1}\right\rfloor(q-1)\right) \\
&=O_{q}\left(\Psi\left(\frac{T}{q-1}\right)\right) .
\end{aligned}
$$

Thus,

$$
\begin{aligned}
\sum_{d=1}^{T} Q(0, \operatorname{gcd}(q-1, d))= & \frac{T}{q-1} \prod_{i=1}^{r}\left(p_{i}^{\left\lfloor\frac{s_{i}}{2}\right\rfloor}+\left(p_{i}-1\right) \sum_{b=1}^{s_{i}} p_{i}^{\left\lfloor\frac{s_{i}+b}{2}\right\rfloor-1}\right) \\
& +O_{q}\left(\Psi\left(\frac{T}{q-1}\right)\right) .
\end{aligned}
$$




\section{Proof of Theorems 1.1-1.2}

Let $N, q \geq 2$, we have

$$
\sum_{n \leq N} d\left(s_{q}\left(n^{2}\right)\right)=\sum_{n \leq N} \sum_{d \mid s_{q}\left(n^{2}\right)} 1=\sum_{d=1}^{(q-1)\left\lfloor\log _{q} q N\right\rfloor} \sum_{\substack{1 \leq n \leq N \\ d \mid s_{q}\left(n^{2}\right)}} 1 .
$$

In view of Lemma 2.1, the last inner sum is $\#\left\{n \leq N: s_{q}\left(n^{2}\right) \equiv 0 \bmod m\right\}$. Thus

$$
\begin{aligned}
\sum_{n \leq N} d\left(s_{q}\left(n^{2}\right)\right) & =\sum_{d=1}^{(q-1)\left\lfloor\log _{q} q N\right\rfloor}\left(\frac{N}{d} Q(0, \operatorname{gcd}(q-1, d))+O\left(N^{1-\sigma_{q, d}}\right)\right) \\
& =N \sum_{d=1}^{(q-1)\left\lfloor\log _{q} q N\right\rfloor} \frac{Q(0, \operatorname{gcd}(q-1, d))}{d}+\sum_{d=1}^{(q-1)\left\lfloor\log _{q} q N\right\rfloor} O\left(N^{1-\sigma_{q, d}}\right) .
\end{aligned}
$$

Using the partial summation and Lemma 2.3, we have

$$
\begin{aligned}
& \sum_{d=1}^{(q-1)\left\lfloor\log _{q} q N\right\rfloor} \frac{Q(0, \operatorname{gcd}(q-1, d))}{d} \\
& =\frac{1}{q-1} \prod_{i=1}^{r}\left(p_{i}^{\left\lfloor\frac{s_{i}}{2}\right\rfloor}+\left(p_{i}-1\right) \sum_{b=1}^{s_{i}} p_{i}^{\left\lfloor\frac{s_{i}+b}{2}\right\rfloor-1}\right) \\
& +\int_{1}^{(q-1)\left\lfloor\log _{q} q N\right\rfloor}\left(\sum_{d=1}^{t} Q(0, \operatorname{gcd}(q-1, d))\right) \frac{d t}{t^{2}} \\
& =\frac{1}{q-1} \prod_{i=1}^{r}\left(p_{i}^{\left\lfloor\frac{s_{i}}{2}\right\rfloor}+\left(p_{i}-1\right) \sum_{b=1}^{s_{i}} p_{i}^{\left\lfloor\frac{s_{i}+b}{2}\right\rfloor-1}\right) \\
& +\int_{1}^{(q-1)\left\lfloor\log _{q} q N\right\rfloor}\left(\frac{t}{q-1} \prod_{i=1}^{r}\left(p_{i}^{\left\lfloor\frac{s_{i}}{2}\right\rfloor}+\left(p_{i}-1\right) \sum_{b=1}^{s_{i}} p_{i}^{\left\lfloor\frac{s_{i}+b-2}{2}\right\rfloor}\right)\right) \frac{d t}{t^{2}} \\
& +\int_{1}^{(q-1)\left\lfloor\log _{q} q N\right\rfloor} O_{q}\left(\Psi\left(\frac{t}{q-1}\right)\right) \frac{d t}{t^{2}} \\
& =\frac{1}{q-1} \prod_{i=1}^{r}\left(p_{i}^{\left\lfloor\frac{s_{i}}{2}\right\rfloor}+\left(p_{i}-1\right) \sum_{b=1}^{s_{i}} p_{i}^{\left\lfloor\frac{s_{i}+b}{2}\right\rfloor-1}\right) \\
& +\frac{1}{q-1} \ln \left((q-1)\left\lfloor\log _{q} q N\right\rfloor\right) \prod_{i=1}^{r}\left(p_{i}^{\left\lfloor\frac{s_{i}}{2}\right\rfloor}+\left(p_{i}-1\right) \sum_{b=1}^{s_{i}} p_{i}^{\left\lfloor\frac{s_{i}+b}{2}\right\rfloor-1}\right) \\
& +O_{q}\left((\log N)^{-1+\epsilon}\right) \\
& =\frac{1+\ln \left((q-1)\left\lfloor\log _{q} q N\right\rfloor\right)}{q-1} \prod_{i=1}^{r}\left(p_{i}^{\left\lfloor\frac{s_{i}}{2}\right\rfloor}+\left(p_{i}-1\right) \sum_{b=1}^{s_{i}} p_{i}^{\left\lfloor\left\lfloor\frac{s}{i}+b^{2}\right\rfloor-1\right.}\right) \\
& +O_{q}\left((\log N)^{-1+\varepsilon}\right), \quad \varepsilon>0 .
\end{aligned}
$$

Inserting this to (3.1), we have 


$$
\begin{aligned}
\sum_{n \leq N} d\left(s_{q}\left(n^{2}\right)\right)= & \frac{1+\ln \left((q-1)\left\lfloor\log _{q} q N\right\rfloor\right)}{q-1} \prod_{i=1}^{r}\left(p_{i}^{\left\lfloor\frac{s_{i}}{2}\right\rfloor}+\left(p_{i}-1\right) \sum_{b=1}^{s_{i}} p_{i}^{\left\lfloor\frac{s_{i}+b}{2}\right\rfloor-1}\right) N \\
& +O_{q}\left(N(\log N)^{-1+\epsilon}\right)+\sum_{d=1}^{(q-1)\left\lfloor\log _{q} q N\right\rfloor} O\left(N^{1-\sigma_{q, d}}\right) \\
= & \frac{1+\ln \left((q-1)\left\lfloor\log _{q} q N\right\rfloor\right)}{q-1} \prod_{i=1}^{r}\left(p_{i}^{\left\lfloor\frac{s_{i}}{2}\right\rfloor}+\left(p_{i}-1\right) \sum_{b=1}^{s_{i}} p_{i}^{\left\lfloor\frac{s_{i}+b}{2}\right\rfloor-1}\right) N \\
& +O_{q}\left(N(\log N)^{-1+\varepsilon}\right), \quad \varepsilon>0 .
\end{aligned}
$$

\section{Average number of divisors of the sum of digits of power $h, h \geq 2$}

In this section, we extend Theorem 1.1 to the sum of digits of power $h, h \geq 2$. To this, we need the following result of M. Drmota, C. Mauduit and J. Rivat [2] about the sum of digits function of polynomial sequences.

Lemma 4.1. [2, Theorem 2] For a fixed polynomail $p(x)=a_{h} x^{h}+\cdots+a_{0} \in \mathbb{Z}$ of degree $h \geq 2$ with $p(\mathbb{N}) \subset \mathbb{N}$. Let $q \geq \exp \left(67 h^{3}(\log h)^{2}\right)$ be a sufficiently large prime number and suppose $\left(a_{h}, q\right)=1$. Then there exists $\sigma_{q, m}>0$ such that for any $y \in \mathbb{Z}$, as $N \rightarrow \infty$,

$$
\#\left\{0 \leq n<N: s_{q}(p(x)) \equiv g \bmod m\right\}=\frac{N}{m} Q^{*}(g, d)+O_{q, m, p}\left(N^{1-\sigma_{q, m}}\right),
$$

where $d=\operatorname{gcd}(m, q-1)$ and

$$
Q^{*}(g, d)=\#\{0 \leq n<d: p(x) \equiv g \bmod d\} .
$$

For our situation, we put $p(x)$ by $x^{h}$ and $g=0$ in Lemma 4.1. We let

$$
Q_{h}^{*}(0, d)=\#\left\{0 \leq n<d: x^{h} \equiv 0 \bmod d\right\} .
$$

We see that, $Q_{h}^{*}\left(0, p^{k}\right)=p^{\lfloor(h-1) k / h\rfloor}$. Thus it is obvious that the function $Q_{h}^{*}(0,$.$) is multiplicative.$ Applying Lemma 2.2, we have following Lemma.

Lemma 4.2. Let a positive integer $h \geq 2$ and $q \geq \exp \left(67 h^{3}(\log h)^{2}\right)$ be a sufficiently large prime number. We write $q-1=\prod_{i=1}^{r} p_{i}^{s_{i}}$.

For $T>1$, if $q-1 \backslash$, then

$$
\begin{aligned}
\sum_{d=1}^{T} Q_{h}^{*}(0, g c d(q-1, d))= & \frac{T}{q-1} \prod_{i=1}^{r}\left(p_{i}^{\left\lfloor\frac{s_{i}(h-1)}{h}\right\rfloor}+\left(p_{i}-1\right) \sum_{b=1}^{s_{i}} p_{i}^{\left\lfloor\frac{s_{i}+b}{h}\right\rfloor-1}\right) \\
& +O_{q}\left(\Psi\left(\frac{T}{q-1}\right)\right) .
\end{aligned}
$$

If $q-1 \mid T$, then

$$
\sum_{d=1}^{T} Q_{h}^{*}(0, \operatorname{gcd}(q-1, d))=\frac{T}{q-1} \prod_{i=1}^{r}\left(p_{i}^{\left\lfloor\frac{s_{i}(h-1)}{h}\right\rfloor}+\left(p_{i}-1\right) \sum_{b=1}^{s_{i}} p_{i}^{\left\lfloor\frac{s_{i}+b}{h}\right\rfloor-1}\right) .
$$


In view of Lemma 4.1 and 4.2 , the proof of Theorem 1.2 is similar to the proof in Theorem 1.1.

\section{Conclusions}

Theorems 1.1-1.2 indicated that $\sum_{n \leq N} d\left(s_{q}\left(n^{h}\right)\right) \ll_{q, h} N \ln (\log N)$. This is a new view of the asymptotic behaviour of the sum (1.1). Moreover, the results showed that the constant depends on the degree of the polynomial and the base $q$.

However, the results should be extended to general polynomials $p(x) \in \mathbb{Z}[x]$. To this we need to know the estimation of the sum $\sum_{d \leq T} Q_{h}^{*}(0, \operatorname{gcd}(q-1, d))$, where

$$
Q_{h}^{*}(0, D)=\#\{0 \leq n<D \mid p(n) \equiv 0 \quad(\bmod D)\} .
$$

\section{Acknowledgements}

We would like to thank the referee for his/her comments and suggestions. Part of this work is supported by the Thailand research fund under Grant No. MRG 6080210.

\section{References}

[1] Erdös, P. (1952) On the sum $\sum d(f(k))$. Journal of the London Mathematical Society, 1.1, $7-15$.

[2] Drmota, M., Mauduit, C. \& Rivat, J. (2011) The sum of digits function of polynomial sequences. Journal of the London Mathematical Society 84.1, 81-102.

[3] Holleboom, L. J. (2014) Prime number decomposition of the Fourier transform of a function of the greatest common divisor. arXiv preprint arXiv:1410.2054.

[4] Hooley, C. (1963) On the number of divisors of quadratic polynomials. Acta Mathematica, 110.1, 97-114.

[5] Lapkova, K. (2016) Explicit upper bound for an average number of divisors of quadratic polynomials. Archiv der Mathematik, 106.3 , 247-256.

[6] Mauduit, C. \& Rivat, J. (2009) La somme des chiffres des carrs. Acta Mathematica, 203.1, 107-148.

[7] Sándor, J. \& Crstici, B. (2004) Handbook of Number Theory II. Kluwer Academic, Dordrecht.

[8] Sándor, J., Mitrinovic, D.S. \& Crstici, B. (2006) Handbook of Number Theory I. Springer, Netherlands. 